\title{
Oral and Pharyngeal Cancer Control and Early Detection
}

\author{
Sol Silverman Jr • A. Ross Kerr • Joel B. Epstein
}

Received: 28 October 2009 / Accepted: 8 January 2010/Published online: 5 March 2010

(C) The Author(s) 2010. This article is published with open access at Springerlink.com

\begin{abstract}
Sixty-four standardized continuing education courses were given for dentists throughout the ten public health districts of the USA to determine if certain behaviors regarding oral and pharyngeal cancer (OPC) control could be modified. Questionnaires were obtained at baseline and at 6 months along with matched control groups. One thousand eight hundred two general dentists participated at baseline and 988 at a 6-month questionnaire follow-up. Analysis of the data indicated that continuing education courses had a positive influence on participants' oral cancer attitudes, knowledge, and behavior that potentially could make a difference on prevention, early detection, and ultimately OPC control.
\end{abstract}

Keywords Oral cancer Early detection .

Continuing education $\cdot$ Prevention

S. Silverman $\operatorname{Jr}(\square)$

Department Orofacial Sciences, School of Dentistry Box 0422,

University of California,

513 Parnassus Avenue,

San Francisco, CA 94143, USA

e-mail: silvermans@dentistry.ucsf.edu

\section{A. R. Kerr}

Department of Oral \& Maxillofacial Pathology,

Radiology \& Medicine, New York University College of Dentistry,

345 E. 24th Street,

New York, NY 10010, USA

e-mail: ark3@NYU.edu

\section{J. B. Epstein}

Department of Oral Medicine,

University of Illinois College of Dentistry,

801 S. Paulina Street,

Chicago, IL 60612, USA

e-mail: jepstein@UIC.edu

\section{Background}

In the USA, oral and pharyngeal cancer (OPC) ranks as the 4th leading cancer site in black men, and the 6th most common site in white men. OPCs are twice as frequent in men as compared with women and currently are estimated to occur in over 35,000 Americans each year, which is an increase over the three previous years [1]. While OPCs occur more commonly in individuals over the age of 45 , particularly in those that smoke and drink alcohol, OPC can occur in persons with no evident risk habits and who are under 40. The recent increase in OPC may be in part associated with human papilloma virus(es). Racial disparities are known, with both OPC rates and mortality higher in African Americans than any other racial group [2].

Despite the advances in surgery, radiation, chemotherapy, and targeted-therapy, cure rates and survival remain poor, with the 5-year survival rate approximating 60\%. This reflects the problem that the majority of OPC's are advanced tumors at the time of diagnosis and treatment. However, if these malignancies are diagnosed and treated in the early stages, the survival rate exceeds $80 \%$.

Carcinogenesis is influenced both by inherited and/or somatic genetic mutations, the latter accumulated principally from exposure to the major risk factors for OPCs, namely tobacco and heavy alcohol use. A distinct subset of OPCs are associated with oncogenic human papilloma viruses (mainly Type 16). This involves different transmission factors and suggests new directions in approaches to prevention and treatment [3]. Nutrition plays a role, albeit may be minor. Diets high in vegetables and fruits have shown to be of some benefit [4].

Prevention is critically important through early detection and tobacco-use cessation. The biology, risks and cessation approaches related to tobacco use are assessed in the paper 
on "tobacco cessation education for dentists." Recognizing and managing premalignant lesions, primarily the leukoplakias and erythroleukoplakias, may prevent some malignant transformations [5-7]. However, in analyzing all experiences and reports, at present, it is clear that early diagnosis is critically essential and may have the most impact for improving survival and cure rates as well as reducing morbidity and cost and improving the quality of life in OPC patients.

The challenge for early diagnosis emphasizes our responsibilities in public and professional education regarding periodic oral cancer examinations for detecting premalignant lesions and early cancer. In this respect, our standard for assessments remains detection during visual/tactile examination, followed by tissue biopsy and microscopic diagnosis. The role and value of chairside adjunctive techniques in the hands of practicing general dentists to facilitate the detection of lesions with malignant potential remains unknown. Current molecular markers for identifying tissue/cells with malignant potential lack specificity and availability. Therefore, degrees of dysplasia as assessed histologically, in spite of controversies regarding interpretation and significance, remain our best indicator of risk for malignant transformation of premalignant oral lesions. Research will eventually resolve this problem and assist in prevention and treatment approaches.

At present, more aggressive treatment combinations and extending conventional limits of therapy have been utilized to increase survival rates. This results in increased cost of care and increased lifelong morbidity. Dental professionals serve an important role as part of the treatment team by assessing pre-treatment oral health to minimize complications of therapy, assisting with acute and chronic side effects of therapy, and participating in rehabilitation to assist patient return to family and society with an acceptable quality-of-life. Oral care runs the full spectrum of patient survivorship.

\section{Results}

A total of 1,892 general dentists in private practice attended one of the continuing education (CE) programs. Of these attendees, 988 (52.2\%) completed the questionnaire 6 months later. Of the 1,015 controls completing the questionnaire at baseline, $678(66.8 \%)$ also completed it at 6 months. Demographics/background variables did not significantly differ between groups at baseline or with the random sample of dentists, therefore suggesting generalizability of the results to the US dentist population. Attrition from the study did not significantly shift the demographics/background variables of the attendee and control groups, indicating there was no bias due to non-response.

There were a number of interesting findings relating to the detection of OPC.
In terms of attitude (both at baseline and 6 month timepoints), $>95 \%$ of all groups recognized the importance of OPC detection. Despite attitude, in terms of knowledge, only $82.6 \%$ indicated at baseline that they understood the steps comprising OPC screening (86.7\% in controls). However, there was a significant improvement to $92.7 \%$ for the attendees at the 6 month follow-up $(p<.001)$, with no improvement in controls. Younger dentists were more knowledgeable.

In terms of behavior (office practices), $>90 \%$ of both attendees and controls stated that they performed OPC examinations, and younger dentists were more likely to perform OPC screenings than their older colleagues. The inclusion of neck palpation as part of the examination significantly increased from $60 \%$ at baseline to $69.1 \%$ in the 6 month follow-up (p.001), with a smaller and insignificant improvement in controls. Stratifying those to be examined by patient age revealed that at baseline both groups reported screening a greater percentage of patients over $40(>90 \%)$ compared with under 40 . However, at 6 months, the attendees compared with controls reported screening a significantly higher percentage of patients, regardless of age, suggesting that taking the module influenced attendees to screen a broader age range. Furthermore, OPC screenings at recall visits increased an average of $2 \%$ over initial visit examinations for all age groups.

Adjunctive diagnostic techniques were not commonly used by attendees or controls either at baseline or at 6 months. When asked how many patients in the last 12 months they had either biopsied or referred, attendees responded about six or seven patients on average. This was identical to the control group and showed no significant changes at 6 months.

Across all groups, only approximately $70 \%$ indicated that they informed patients when they were performing an OPC examination. This is of concern, since this information is important in conveying public OPC awareness. Our finding is consistent with studies that have shown low patient awareness of OPC [8-10] as well as the importance of OPC examinations.

Multivariate models of the outcome variables comparing attendees supported bivariate analysis and indicated that attendees gained knowledge and a tendency to modify their practice behavior.

\section{Conclusions and Comments}

There were a number of limitations to this study. First, the short follow-up period does not predict a possible lapse in knowledge and behaviors over time. This must be assessed by a longer term follow-up with larger groups of dental practitioners. The impact of time on retention of knowledge and behavioral change is suggested in a recent survey of a 
dental practice group [10]. Second, due to the high response rates to many of the questions, there was a "ceiling effect," which made it difficult to assess differences between the groups. Questionnaire design and field testing is of paramount importance to minimize this effect.

Despite limitations, the CE module on early detection of OPC showed an overall benefit to attendees in terms of improvement in the aforementioned areas of knowledge, attitudes and clinical practices. This is an important finding showing that continuing education impacts not only knowledge and attitudes, but also may modify behaviors of dental practitioners.

It is evident that our most effective control of OPC at present depends upon early detection by identifying oral mucosal changes through adequate oral cancer examinations, with confirmation by biopsy the presence or absence of premalignant lesions and cancer. Professional education must be emphasized as well as public education. This will raise awareness and minimize delays in diagnosis. Prevention, treatment and rehabilitation of OPC are all a part of dental/oral health care. In this way, dentistry can effectively play a role in OPC control and make a difference!

Open Access This article is distributed under the terms of the Creative Commons Attribution Noncommercial License which permits any noncommercial use, distribution, and reproduction in any medium, provided the original author(s) and source are credited.

\section{References}

1. Jemal A, Siegel R, Ward E et al (2009) Cancer statistics, 2009. CA Cancer J Clin 59:225-249

2. Molina MA, Cheung MC, Perez EA et al (2008) African American and poor patients have a dramatically worse prognosis for head and neck cancer. An examination of 20,915 patients. Cancer 113:2797-2806

3. Hennessey PT, Westra WH, Califano JA (2009) Human papillomavirus and head and neck squamous cell carcinoma: recent evidence and clinical implications. J Dent Res 88:300-306

4. Chainini-Wu N (2002) Diet and oral, pharyngeal, and esophageal cancer. Nutr Cancer 44:1040126

5. Silverman S Jr (1998) Diagnosis and management of leukoplakia and premalignant lesions. Oral Maxillofac Surg Clin N Amer 10:13-23

6. Schoelch ML, Sekandari N, Regezi JA, Silverman S Jr (1999) Laser management of oral leukoplakias: a follow-up study of 70 patients. Laryngoscope 109:949-953

7. van der Waal I (2009) Potentially malignant disorders of the oral and oropharyngeal mucosa; terminology, classification and present concepts of management. Oral Oncol 45:317323

8. Horowitz AM, Canto MT, Child WL (2002) Maryland adults' perspectives on oral cancer prevention and early detection. JADA 133:1058-1071

9. National Center Health Statistics (2009) Percentage of adults aged $>18$ years who have ever had an oral cancer examination, by smoking status and age group - National Health Interview Survey, United States, 2008. MMWR 58:1013

10. LeHew CW, Epstein JB, Kaste LM, Cho Y-K (2009) Assessing oral cancer early detection: clarifying dentist practices. J Pub $\mathrm{H}$ Dent (ISSN 0022-4006), Published online 